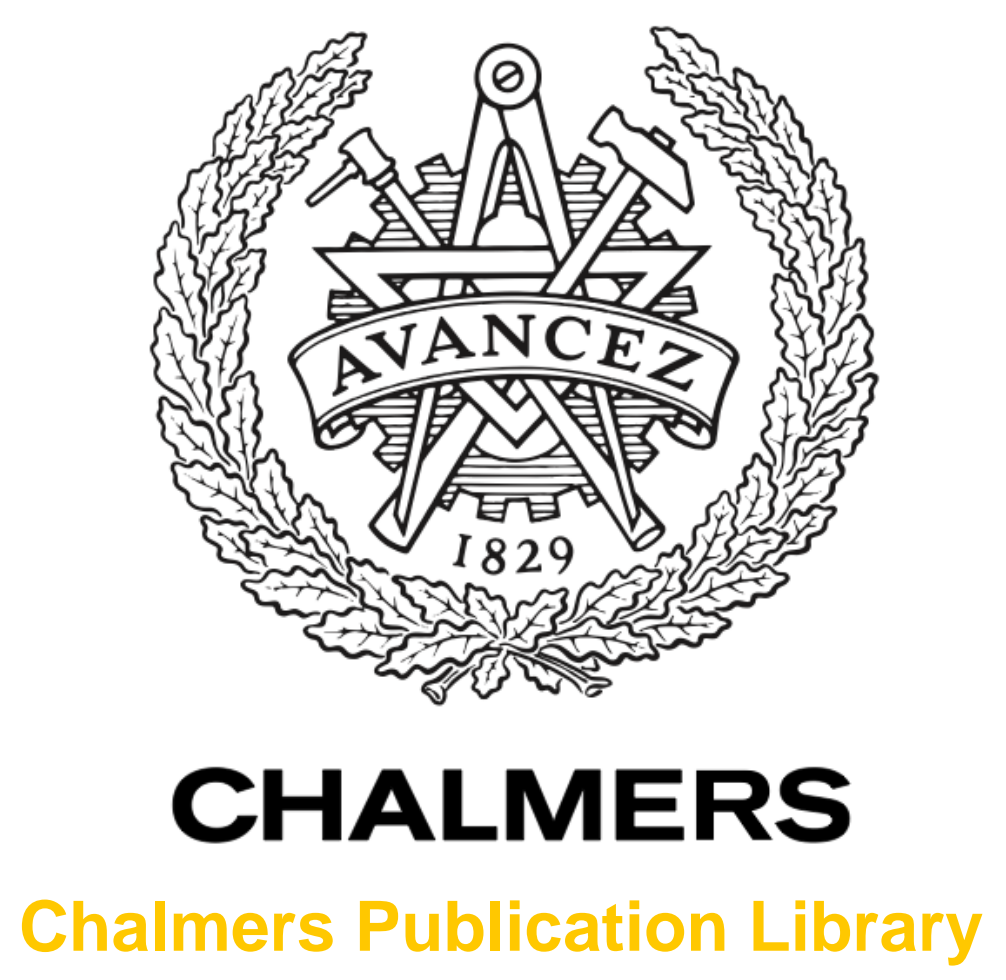

\author{
On the Fully Automatic Construction of a Realistic Head Model for EEG Source \\ Localization
}

This document has been downloaded from Chalmers Publication Library $(\mathrm{CPL})$. It is the author's version of a work that was accepted for publication in:

i Proceedings of the Annual International Conference of the IEEE Engineering in Medicine and Biology Society, EMBS. Osaka; Japan; 3 July 2013 through 7 July 2013 (ISSN: 1557170X)

Citation for the published paper:

Mahmood, Q. ; Shirvany, Y. ; Chodorowski, A. (2013) "On the Fully Automatic

Construction of a Realistic Head Model for EEG Source Localization". i Proceedings of the Annual International Conference of the IEEE Engineering in Medicine and Biology Society, EMBS. Osaka; Japan; 3 July 2013 through 7 July 2013 pp. 3331-3334.

http://dx.doi.org/10.1109/EMBC.2013.6610254

Downloaded from: http://publications.lib.chalmers.se/publication/185904

Notice: Changes introduced as a result of publishing processes such as copy-editing and formatting may not be reflected in this document. For a definitive version of this work, please refer to the published source. Please note that access to the published version might require a subscription. 


\title{
On the Fully Automatic Construction of a Realistic Head Model for EEG Source Localization
}

\author{
Qaiser Mahmood, ${ }^{1}$ Yazdan Shirvany, ${ }^{1}$ Andrew Mehnert, ${ }^{1}$ Artur Chodorowski, ${ }^{1}$ Johanna Gellermann, ${ }^{2}$ \\ Fredrik Edelvik, ${ }^{3}$ Anders Hedström, ${ }^{4}$ Mikael Persson ${ }^{1}$
}

\begin{abstract}
Accurate multi-tissue segmentation of magnetic resonance (MR) images is an essential first step in the construction of a realistic finite element head conductivity model (FEHCM) for electroencephalography (EEG) source localization. All of the segmentation approaches proposed to date for this purpose require manual intervention or correction and are thus laborious, time-consuming, and subjective. In this paper we propose and evaluate a fully automatic method based on a hierarchical segmentation approach (HSA) incorporating Bayesian-based adaptive mean-shift segmentation (BAMS). An evaluation of HSA-BAMS, as well as two reference methods, in terms of both segmentation accuracy and the source localization accuracy of the resulting FEHCM is also presented. The evaluation was performed using (i) synthetic 2D multimodal MRI head data and synthetic EEG (generated for a prescribed source), and (ii) real 3D T1-weighted MRI head data and real EEG data (with expert determined source localization). Expert manual segmentation served as segmentation ground truth. The results show that HSA-BAMS outperforms the two reference methods and that it can be used as a surrogate for manual segmentation for the construction of a realistic FEHCM for EEG source localization.
\end{abstract}

\section{INTRODUCTION}

Electroencephalography (EEG) source localization is a tool used to locate the source of epileptic seizures in the brain. In the case of focal seizures that cannot be satisfactorily treated by medication this information is used to guide surgical resection of the abnormal tissue. Accurate localization is thus essential. The accuracy is determined not only by the methods used to solve the underlying forward and inverse problems but also the quality and fidelity of the patient-specific head conductivity model used. This paper focuses the latter.

The construction of a realistic head conductivity model involves, as a first step, the accurate segmentation of the patient's head tissues from magnetic resonance (MR) images. The segmentation can then be used to construct a finite element model in which each tissue type is assigned known conductivity properties. The segmentation

${ }^{1}$ Q. Mahmood, Y. Shirvany, A. Mehnert, A. Chodorowski, M. Persson are with the Department of Signals and Systems, Chalmers University of Technology and MedTech West Center, Göteborg, Sweden (e-mail: qaiserm@chalmers.se).

${ }^{2} \mathrm{~J}$. Gellermann is with the Universitätsklinik für Radioonkologie, Tübingen, Germany.

${ }^{3}$ F. Edelvik is with the Fraunhofer-Chalmers Research Center, Chalmers Science Park, Göteborg, Sweden.

${ }^{4}$ A. Hedström is with the Sahlgrenska Academy, Göteborg, Sweden. approaches proposed to date for this purpose [1-3] all require manual intervention. Consequently the segmentation process is laborious, time-consuming, and subjective. A fully automatic method is thus highly desirable.

We recently proposed a fully automatic multi-tissue segmentation method for multi-modal MRI images of the head [4]. The method is based on a hierarchical segmentation approach (HSA) incorporating Bayesian-based adaptive mean-shift segmentation (BAMS). In this paper we propose the use of HSA-BAMS for the fully automatic construction of a patient-specific realistic finite element head conductivity model (FEHCM) for use in EEG source localization. We present an evaluation of the performance of HSA-BAMS, as well as that of two reference methods, using both synthetic 2D multi-modal MRI head data and real 3D T1-weighted MRI head data with corresponding segmentation ground truth (expert manual segmentation). The first reference method is BET-FAST [5, 6] commonly used in the creation of patient specific conductivity models [7]. The second is an instantiation of the HSA incorporating the HMRF-EM (hidden Markov random field model and associated Expectation-Maximization) algorithm [6] implemented in the FAST tool in FSL.

\section{METHOD}

\section{A. MRI Data}

We used two different data sets to evaluate the performance of our proposed HSA-BAMS method for EEG source localization. Data set 1 originates from synthetic T1-, T2-, and (proton density) PD-weighted MRI scans of a human head, with $1 \%$ Gaussian noise, from the Brainweb simulated brain database (SBD) [8]. Data set 1 consists only of slice number 100 from each scan (spatially co-registered). The slice comprises $181 \times 217$ pixels of size $1 \mathrm{~mm}^{2}$. Data set 2 comprises a T1-weighted MRI volume of the head of a healthy subject acquired on a 3T Philips Achieva scanner using a gradient echo sequence with the following parameters: $\mathrm{TR}=8.1655 \mathrm{msec}, \mathrm{TE}=3.7570 \mathrm{msec}$, flip angle of $8^{\circ}$, and an acquisition matrix of size $256 \times 256 \times 195$. The size of each voxel is $0.9375 \mathrm{~mm}^{3}$.

The ground truth (GT) segmentation for data set 1 was obtained from the 9 tissue labels in Brainweb. These were reduced to 5 labels by merging the connective, fat, muscle and skin tissue classes, and the glial matter and gray matter 
classes. The GT for data set 2 was obtained from a manual segmentation of the volume by an experienced radiooncologist (J.G.) into five tissues: white matter (WM), gray matter (GM), cerebrospinal fluid (CSF), skin and skull/bone.

\section{B. Proposed Hierarchical Segmentation Approach (HSA)}

A schematic of the HSA is presented in Fig.1. The HSA conceptually takes as input a volume $(V)$ comprising vectorvalued voxels (each component originates from a different MRI scan). This volume is segmented into two disjoint subvolumes, brain tissue $\left(V_{\mathrm{BT}}\right)$ and non-brain-tissue $\left(V_{\mathrm{NBT}}\right)$, using a brain-tissue segmentation algorithm (BTSA) and a non-brain-tissue segmentation algorithm (NBTSA) respectively. In the experiments below we used BET [5] as the BTSA and a simple algorithm based on thresholding [9] and morphological reconstruction [10] for the NBTSA. Finally a multi-tissue segmentation algorithm (MTSA) is applied independently to the $V_{\mathrm{BT}}$ and $V_{\mathrm{NBT}}$ volumes to segment them into individual tissue classes WM, GM, CSF and skin, skull/bone respectively. In the experiments below we used the following MTSAs: our own BAMS and the HMRF-EM method implemented in FAST.

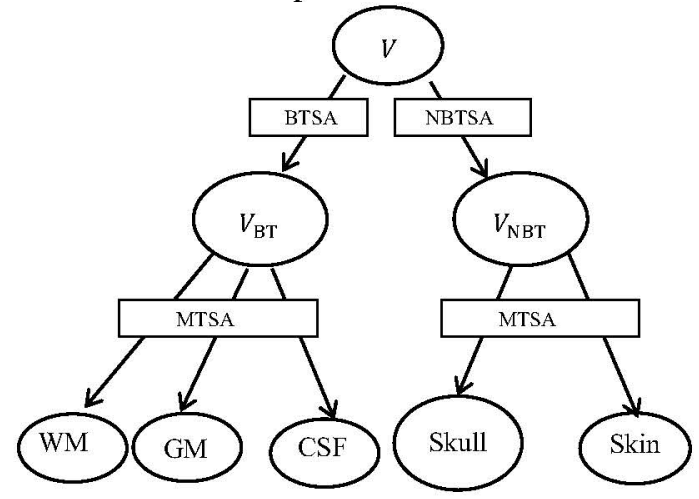

Figure 1. Schematic of the proposed HSA for automated whole head segmentation. See the text for a description of the acronyms and variables.

\section{Proposed MTSA; Bayesian Adaptive Mean Shift (BAMS)}

Our BAMS segmentation algorithm is based on the adaptive mean shift (AMS) segmentation framework proposed by Mayer and Greenspan [11]. The reader is referred to [11] for explanation of AMS framework. In BAMS, the $k \mathrm{NN}$ adaptive bandwidth estimator [11] is replaced with our own Bayesian adaptive bandwidth estimator. This estimator is in turn a novel variation on the Bayesian fixed bandwidth estimator proposed in [12]. The bandwidth is modeled by the a posteriori probability density function $p(s \mid \mathbf{x})$ of local data spread $s$ given the data point $\mathbf{x}$. Let $M<n$ (total number of data pints) be the number of nearest neighborhoods to a data point $\mathbf{x}_{i}$. We can then define the pseudolikelihood

$$
P(s \mid \mathbf{x})=\prod_{j=1}^{N} P\left(s \mid \mathbf{x}_{M_{j}}\right)
$$

where $P\left(s \mid \mathbf{x}_{M_{j}}\right)$ is the probability of local data spread $s$ based on the $M_{j}$ nearest neighbors data samples to $\mathbf{x}_{M_{j}}$ and
$\left\{M_{j}, j=1, \ldots, N\right\}$ is the set of $N$ neighborhoods of various sizes. The evaluation of probabilities over the entire range of $M_{j}$ is expressed as

$$
P\left(s \mid \mathbf{x}_{M_{j}}\right)=\int P\left(s \mid M_{j}, \mathbf{x}_{M_{j}}\right) P\left(M_{j} \mid \mathbf{x}_{M_{j}}\right) d M_{j}
$$

Applying Bayes rule we get

$$
P\left(M_{j} \mid \mathbf{x}_{M_{j}}\right)=\frac{P\left(\mathbf{x}_{M_{j}} \mid M_{j}\right) P\left(M_{j}\right)}{P\left(\mathbf{x}_{M_{j}}\right)}
$$

where $P\left(\mathbf{x}_{M_{j}} \mid M_{j}\right)$ is the probability of the data point $\mathbf{x}_{M_{j}}$ given the $M_{j}$ nearest neighborhood. Hereinafter $P\left(M_{j}\right)$ is considered to be a uniform distribution on the interval $\left[M_{1}, M_{2}\right]$. Several values are selected for $M_{j}$ in this interval according to

$$
M_{j}=M_{1}+j \frac{M_{2}-M_{1}}{N}
$$

For a given $M_{j}$ the local variance $s_{j}$ is computed as

$$
s_{j}=\frac{\sum_{l=1}^{M_{j}}\left\|\mathbf{x}_{(i, l)}-\mathbf{x}_{i}\right\|^{2}}{M_{j}-1}, i=1,2 \ldots . n, j=1,2, \ldots N
$$

where $\mathbf{x}_{(i, l)}$ is the nearest neighbor to the feature point $\mathbf{x}_{i}$. The distribution of variances is modeled as a Gamma distribution. Finally the adaptive bandwidth is computed as the mean of the Gamma distribution and is given by

$$
\widehat{h}\left(\mathbf{x}_{i}\right)=\hat{\alpha} \hat{\beta}, i=1,2, \ldots n
$$

\section{EEG Data}

Synthetic EEG was generated for data set 1 by placing a source in the GM of the GT image and calculating EEG signals from 30 electrodes placed equidistantly around the model. The real EEG data for data set 2 was obtained by recording the somatosensory evoked potentials (SEPs) on the subject's scalp. These SEPs were generated by stimulating the left wrist median nerve by electric pulses and EEG measurement was done using 61 electrodes based on 10/10 system [13]. The GT for the source was taken to be the expected source region determined independently by an experienced clinician (A.H.) based on neurophysiological knowledge.

\section{E. EEG Source Localization}

Given a FEHCM the EEG source localization procedure involves the solution of the following two problems: (i) the forward problem which deals with finding the scalp potentials for the given current sources and (ii) the inverse problem which deals with estimating the sources to fit the given potential distributions at the scalp electrodes. Herein we used the subtraction method for modeling the dipole in the forward problem [14] and a modified particle swarm optimization (MPSO) [13] method to solve the inverse problem.

\section{F. Evaluation of the Proposed and Reference Segmentation Methods for Source Localization}

HSA-BAMS, HSA-HMRF-EM, and BET-FAST were independently used to segment both data sets. The BET tool threshold parameter ' $f$ ' was set to 0.5 for the extraction 
of $V_{\mathrm{BT}}$. The following parameters were set for HSABAMS: $N=10, M_{1}=100$ and $M_{2}=330$. The parameter MRF beta was set to 0.1 for both the HSA-HMRF-EM and BET-FAST methods. An FEHCM was constructed from each segmentation as well from the GT. Segmentation accuracy was evaluated quantitatively using the Dice index (DI) [15]. EEG source localization performance was evaluated quantitatively using the following metrics: (i) $R E$ (relative error) $=\left\|\mathbf{u}_{\text {meas }}-\mathbf{u}_{\text {est }}\right\| /\left\|\mathbf{u}_{\text {meas }}\right\|$ where $\mathbf{u}_{\text {meas }}$ is a vector of the measured potential on the scalp and $\mathbf{u}_{\text {est }}$ is a vector of the potential generated by the estimated source; (ii) $L E$ (localization error) $=$ $\left\|\mathbf{x}-\mathbf{x}_{\text {est }}\right\|$ where $\mathbf{x}$ is the GT source position and $\mathbf{x}_{e s t}$ is the estimated source position; and (iii) $O E$ (orientation error) $=\cos ^{-1}\left(\frac{\mathbf{M} \cdot \mathbf{M}_{\text {est }}}{\|\mathbf{M}\| \mid \mathbf{M}_{\text {est }} \|}\right)$ where $\mathbf{M}$ is the simulated or GT source dipole moment and $\mathbf{M}_{e s t}$ is the estimated source moment.

\section{REULTS AND Discussion}

\section{A. Segmentation Evaluation}

The DI values for each tissue and each method for both data sets are shown in Table I. They show that the performance of HSA-BAMS is consistently better than that of the two reference methods. Fig. 2 and Fig. 3 show the segmentation results for data set 1 and for a single slice in data set 2 respectively. It can be seen that for HSA-BAMS the results are less noisy and show better delineation for all tissues.

TABLE I. Dice INDEX VALUES For EACH METHOD AND EACH TisSue

\begin{tabular}{|c|c|c|c|c|c|}
\hline \multirow[t]{2}{*}{ Tissue } & \multicolumn{3}{|c|}{ Data Set 1 (2D synthetic) } & \multicolumn{2}{|c|}{ Data Set 2 (3D real) } \\
\hline & $\begin{array}{c}H S A- \\
B A M S\end{array}$ & $\begin{array}{l}\text { BET- } \\
\text { FAST }\end{array}$ & $\begin{array}{c}H S A- \\
H M R F- \\
E M\end{array}$ & $\begin{array}{c}H S A- \\
B A M S\end{array}$ & $\begin{array}{c}H S A- \\
H M R F \\
\text { EM }\end{array}$ \\
\hline WM & 0.991 & 0.905 & 0.905 & 0.885 & 0.879 \\
\hline GM & 0.977 & 0.724 & 0.724 & 0.848 & 0.836 \\
\hline $\mathrm{CSF}$ & 0.953 & 0.635 & 0.635 & 0.523 & 0.517 \\
\hline Skin & 0.958 & 0.956 & 0.815 & 0.868 & 0.822 \\
\hline $\begin{array}{l}\text { Skull/ } \\
\text { Bone }\end{array}$ & 0.981 & 0.886 & 0.880 & 0.697 & 0.588 \\
\hline
\end{tabular}

\section{B. EEG Source Localization Evaluation}

Table II shows that HSA-BAMS has less $R E$ than either of the reference methods. In the case of data set 2 the $R E$ compares favorably to that obtained from the GT segmentation. Table III shows that HSA-BAMS has less $L E$ (a value of zero for data set 1) and less $O E$ than either of the reference methods. Fig. 2(d) shows the estimated source position for data set 1 for each method. Fig. 4 shows the estimated source position for data set 2 for each method and for that obtained using the segmentation GT. It is likely that the poorer localization performance compared to data set 1 is that only T1-weighted data was available for tissue segmentation. Thus both HSA-BAMS and HSA-HMRF-EM were not able to exploit multi-modal data (as was available in data set 1) to achieve more accurate segmentation. Moreover the T1-weighted data in data set 2 has several shortcomings. Firstly the signal intensities for both fat and water are heavily attenuated because of an opposed phase cancelation of signal from both tissues. This leads to skull/bone segmentation errors. Secondly the presence of blood in the upper sagittal sinus means that the intensity of that area changes from low to high. This in turn causes some misclassification of CSF in that area as skin. These errors do not exist in the GT image because the radio-oncologist was able to use her anatomical knowledge to arrive at a more accurate segmentation. This is likely why the EEG source localization obtained from the GT segmentation is more accurate. Overall the results suggest that better quality, and multi-modal, MRI data would greatly improve the accuracy (less $R E$ ) of source localization obtained using HSA-BAMS.

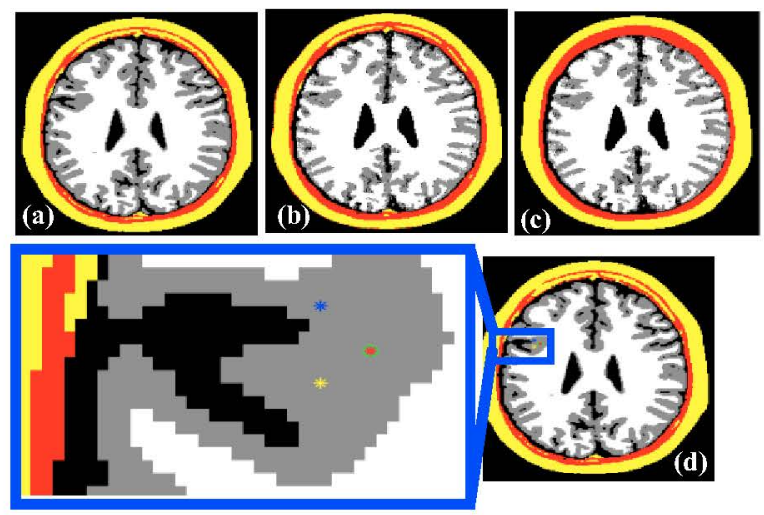

Figure 2. Segmentation and source localization results for data set 1: (a) HSA-BAMS; (b) HSA-HMRF-EM; (c) BET-FAST; and (d) GT superimposed with the location of the simulated (synthetic) source (green), HSA-BAMS estimated source (red), HSA-HMRF-EM source (blue) and BET-FAST source (yellow). WM is white, GM is gray, CSF is black, skull/bone is red and skin is yellow.

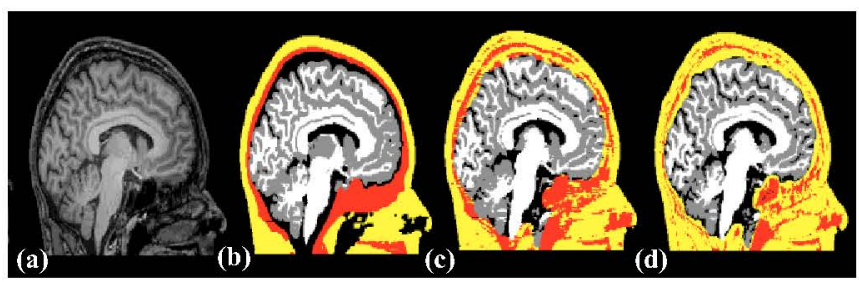

Figure 3. Segmentation results for one sagittal slice from data set 2: (a) Original T1-weighted image; (b) GT; (c) HSA-BAMS; and (d) HSAHMRF-EM (WM is white, GM is gray, CSF is black, skull/bone is red and skin is yellow).

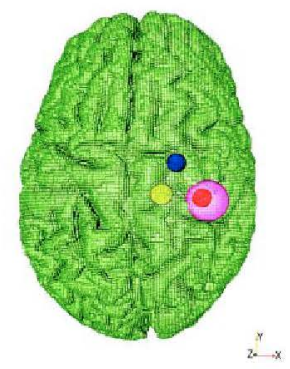

Figure 4. Source localization results for data set 2: from segmentation GT (red); HSA-BAMS (yellow); HSA-HMRF-EM (blue) and clinician marked region (magenta) in the GM. 


\section{Conclusion}

In this work we have presented and evaluated a fully automatic method for the construction of a realistic finite element head conductivity model from multi-modal MRI data. The cornerstone of the method is a fully automatic method for segmenting multiple tissues called HSA-BAMS. It is a hierarchical segmentation approach incorporating Bayesian-based adaptive mean-shift segmentation. We also presented an evaluation of the method, as well as two reference methods, using (i) synthetic $2 \mathrm{D}$ multi-modal MRI head data and synthetic EEG generated for a prescribed source, and (ii) real 3D T1-weighted MRI head data and corresponding real EEG data with expert determined source localization ground truth. Expert manual segmentation served as segmentation ground truth. The results demonstrate the efficacy and accuracy of the method, that it outperforms the two reference methods, and suggest that it can be used as a surrogate for manual segmentation for the construction of a realistic FEHCM for EEG source localization. In the future we aim to evaluate our method with real multi-modal MRI data sets that HSA-BAMS can exploit to achieve more accurate segmentation than possible with the single T1-weigthed data set used in this study.

Table II. RELETIVE ERROR FOR EACH METHOD

\begin{tabular}{|c|c|c|c|}
\hline \multicolumn{2}{|c|}{ Data Set 1 (2D synthetic) } & \multicolumn{2}{c|}{ Data Set 2 (3D real) } \\
\hline Methods & $\boldsymbol{R E}$ & Methods & $\boldsymbol{R} \boldsymbol{E}$ \\
\hline HSA-BAMS & $\mathbf{0 . 0 1}$ & HSA-BAMS & $\mathbf{0 . 3 5}$ \\
\hline HSA-HMRF-EM & 0.03 & HSA-HMRF-EM & 0.42 \\
\hline BET-FAST & 0.20 & Ground truth & $\mathbf{0 . 2 3}$ \\
\hline
\end{tabular}

TABLE III. LOCALIZATION ERROR AND ORIENTATION ERROR

\begin{tabular}{|c|c|c|c|c|}
\hline \multirow{2}{*}{ Methods } & \multicolumn{2}{|c|}{ Data set 1 (2D synthetic) } & \multicolumn{2}{c|}{ Data set 2 (3D real) } \\
\cline { 2 - 5 } & $\boldsymbol{L} \boldsymbol{E}$ & $\boldsymbol{O} \boldsymbol{E}$ & $\boldsymbol{L} \boldsymbol{O}$ & $\boldsymbol{O} \boldsymbol{E}$ \\
\hline HSA-BAMS & $\mathbf{0 . 0 m m}$ & $\mathbf{0 . 0 5}^{\mathbf{0}}$ & $\mathbf{2 1 . 0 m m}$ & $\mathbf{2 2 . 0 ^ { \mathbf { 0 } }}$ \\
\hline $\begin{array}{c}\text { HSA-HMRF- } \\
\text { EM }\end{array}$ & $5.7 \mathrm{~mm}$ & $0.27^{\circ}$ & $25.0 \mathrm{~mm}$ & $24.0^{\circ}$ \\
\hline BET-FAST & $5.1 \mathrm{~mm}$ & $0.18^{\circ}$ & - & - \\
\hline
\end{tabular}

\section{ACKNOWLEDGMENT}

The authors would like to thank Simon Bergstrand for his participation as the healthy subject in this study.

\section{REFERENCES}

[1] M. Rullmann , A. Anwander, M. Dannhauer, S. K. Warfield, F. H. Duffy, C. H. Wolters, "Source EEG source analysis of epileptiform activity using a $1 \mathrm{~mm}$ anisotropic hexahedra finite element head model," Neuroimage, vol. 44, pp. 399-410, 2009.

[2] C. H. Wolters, A. Anwander, X. Tricoche, D. Weinstein, M. A. Koch, R. S. MacLeod "Influence of tissue conductivity anisotropy on EEG/MEG field and return current computation in a realistic head model: a simulation and visualization study using high-resolution finite element modeling," Neuroimage, vol. 30, pp. 813-26, 2006.

[3] Z. A. Acar, S.Makeig, "Neuroelectromagnetic forward modeling toolbox," Journal of Neuroscience Methods, vol. 190, pp. 258-270, 2010.

[4] Q. Mahmood, A. Chodorowski, A. Mehnert, J. Gellermann, M. Persson, "Automatic multitissue segmentation of MR images of the head using a hierarchical Segmentation approach incorporating Bayesian-based adaptive mean-shift," JCAT, submitted for publication.

[5] S. M. Smith, "Fast robust automated brain extraction," Human Brain Mapping, vol. 17, pp. 143-155, 2002.

[6] Y. Zhang, M. Brady, and S. Smith, "Segmentation of brain MR images through a hidden markov random field model and the expectation maximization algorithm," IEEE Trans. Med. Imag., vol. 20, pp. 45-57, 2001.

[7] D. Abhishek, M. B. Julie, M. Bikson, J. Fridriksson, "Individualized model predicts brain current flow during transcranial direct-current stimulation treatment in responsive stroke patient," Brain Stimul, vol.4, pp. 169-174, 2011.

[8] C. A. Cocosco, V. Kollokian, R. K. -S. Kwan, A. C. Evans, "BrainWeb: Online Interface to a 3D MRI Simulated Brain Database," NeuroImage, vol. 5, part 2/4, S425, 1997.

[9] H. Zhang, J. Liu , Z. Zhu, H. Li, "An automated and simple method for brain MR image extraction," BioMedical Engineering Online 2011:10:81.

[10] P. Soille, "Morphological image analysis: principles and applications," Springer-Verlag, 1999.

[11] A. Mayer, H. Greenspan, "An adaptive mean-shift framework for MRI brain segmentation," IEEE Trans. Med. Imag., vol. 28, pp. 1238-1249, 2009.

[12] A. G. Bors, N. Nasios, "Kernel bandwidth estimation for nonparametric modeling," IEEE Trans. SMC, pp. 1543 - 1555, 2009.

[13] Y. Shirvany, F. Edelvik, S. Jakobsson, A. Hedström, Q. Mahmood, A. Chodorowski, M. Persson, "Non-invasive EEG source localization with Particle Swarm Optimization: Clinical Test," in Proc. 34th IEEE Annual Int. Conf. EMBC, 2012, pp. 6232-6235.

[14] F. Edelvik, B. Andersson, S. Jakobsson, S. Larsson, M. Persson, Y. Shirvany, "An improved method for dipole modeling in EEG based source localization," in Proc. IFMBE, 2009, pp.146-149.

[15] L. R. Dice, "Measures of the amount of ecologic association between species," Ecology, vol. 26, pp. 297-302, 1945. 